\title{
Dynamic Phase Transformation during Hot-Forging Process of a Powder Metallurgy $\alpha+\beta$ Titanium Alloy
}

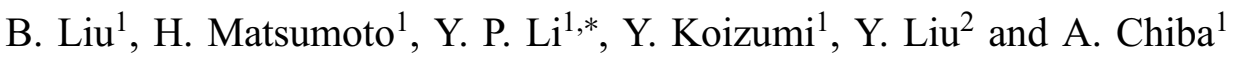 \\ ${ }^{1}$ Institute for Materials Research, Tohoku University, Sendai 980-8577, Japan \\ ${ }^{2}$ State Key Laboratory of Powder Metallurgy, Central South University, Changsha 410083, P. R. China
}

\begin{abstract}
Dynamic phase transformation during subtransus forging was investigated in a powder metallurgy (PM) Ti-1.5Fe-2.25Mo alloy. The results show that compressive deformation restrains the hcp $(\alpha) \rightarrow \operatorname{bcc}(\beta)$ phase transformation, and induces a local martensitic transformation in the deformed sample during subsequent cooling. Thermodynamic analysis confirms the feasibility of these transformations. [doi:10.2320/matertrans.M2012042]
\end{abstract}

(Received February 6, 2012; Accepted February 24, 2012; Published April 25, 2012)

Keywords: titanium alloys, hot deformation, phase transformation, diffusion

\section{Introduction}

Transformation between hcp $(\alpha)$ and bcc $(\beta)$ in titanium alloys could occur during static heat-treatment or dynamic deformation at elevated temperatures, as well as during cooling process. ${ }^{1-3)}$ Several researchers have reported that the transformation during dynamic deformation is different from that during static heat-treatment. For example, Koike et al. $^{4)}$ investigated the dynamic phase transformation in a Ti-5.3Al-1.4Fe (mass\%) alloy under superplastic deformation and found that the $\alpha \rightarrow \beta$ transformation in the $\alpha+\beta$ phase region was significantly enhanced and the $\beta$ transus was reduced by approximate $70 \mathrm{~K}$. Yang et al. ${ }^{5)}$ also found that the tensile deformation could accelerate the $\alpha \rightarrow \beta$ transformation in the Ti-6Al-4V alloy. In addition, an enhanced $\beta \rightarrow \alpha_{2}$ transformation was also reported in a titanium-based $\mathrm{Ti}_{3} \mathrm{Al}$ intermetallic compound. ${ }^{6}$ ) During hot compression, changes in the phase fraction or morphology very frequently occur in titanium alloys, and these changes are assumed to be induced by the temperature. ${ }^{7,8)}$ In the present study, we investigated the dynamic phase transformation during subtransus forging in a PM Ti-1.5Fe-2.25Mo alloy, and provided evidence that the compressive deformation can also significantly alter the $\alpha \rightarrow \beta$ phase transformation. The underlying mechanisms of how the deformation affects the phase transformation were also discussed.

\section{Experimental}

The material used in this study was a PM Ti-1.5Fe$2.25 \mathrm{Mo}$ alloy with chemical composition (in mass $\%$ ) of 1.51 Fe, 2.3 Mo, 0.2 O, 0.05 C, $0.01 \mathrm{~N}$, balance Ti. The materials were sintered at $1573 \mathrm{~K}$ for $3 \mathrm{hrs}$, followed by furnace cooling, resulting in a near full lamellar microstructure with volume fraction of $\alpha$ phase of about $88.5 \%$, as shown in Fig. 1 (this image and all subsequent SEM microstructures were captured via backscattered electron (BSE) imaging). The average colony size is about $65 \mu \mathrm{m}$ and the lamellar spacing is about $1.5 \mu \mathrm{m}$. The $\beta$ transus $\left(T_{\beta}\right)$ was determined to be $1143 \mathrm{~K}$ by metallographic method. Cylindrical speci-

*Corresponding author, E-mail: lyping@imr.tohoku.ac.jp

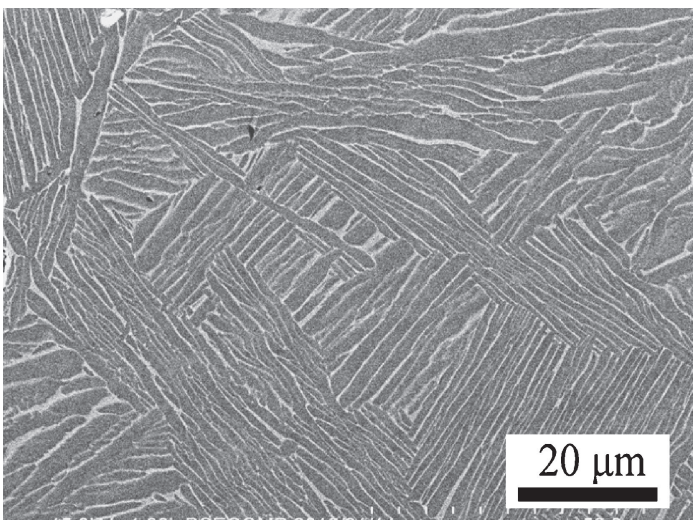

Fig. 1 Microstructure of the sintered $\mathrm{Ti}-1.5 \mathrm{Fe}-2.25 \mathrm{Mo}$ alloy (the light phases are $\beta$ phases and the grey phases are $\alpha$ phases).

mens were cut from the sintered compact for heat treatment and deformation tests. Heat treatment experiments were carried out at $1133 \mathrm{~K}$ to different times. After heat treatment, the samples were quenched by helium gas to room temperature (cooling rate $>100 \mathrm{~K} / \mathrm{s}$ ). Deformation tests were conducted at $1133 \mathrm{~K}$ and strain rate of $0.001 \mathrm{~s}^{-1}$ by employing computer-aided compression equipment (Thermec Master-Z, Fuji Electronic Industrial Co., Ltd., Japan). The deformation specimens were first heated to the deformation temperature at a rate of $3 \mathrm{~K} / \mathrm{s}$, maintained at that temperature for $150 \mathrm{~s}$, and then deformed to the specific strains, finally quenched by helium gas. The microstructures of the heat-treated and deformed samples were characterized by scanning electron microscopy (SEM), Transmission electron microscopy (TEM), electron probe microanalysis (EPMA), and X-ray diffraction (XRD).

\section{Results and Discussions}

Figures 2(a) and 2(b) show the microstructures of the Ti$1.5 \mathrm{Fe}-2.25 \mathrm{Mo}$ alloy heat-treated at $1133 \mathrm{~K}$ for 1 and $3.5 \mathrm{ks}$, respectively. The heat-treated microstructures consist of primary $\alpha$ phase and acicular $\alpha^{\prime}$ martensite (grey areas, hereafter referred to as transformed $\beta$ phase). The volume fraction of primary $\alpha$ phase decreases from 11 to $3 \%$ as the 

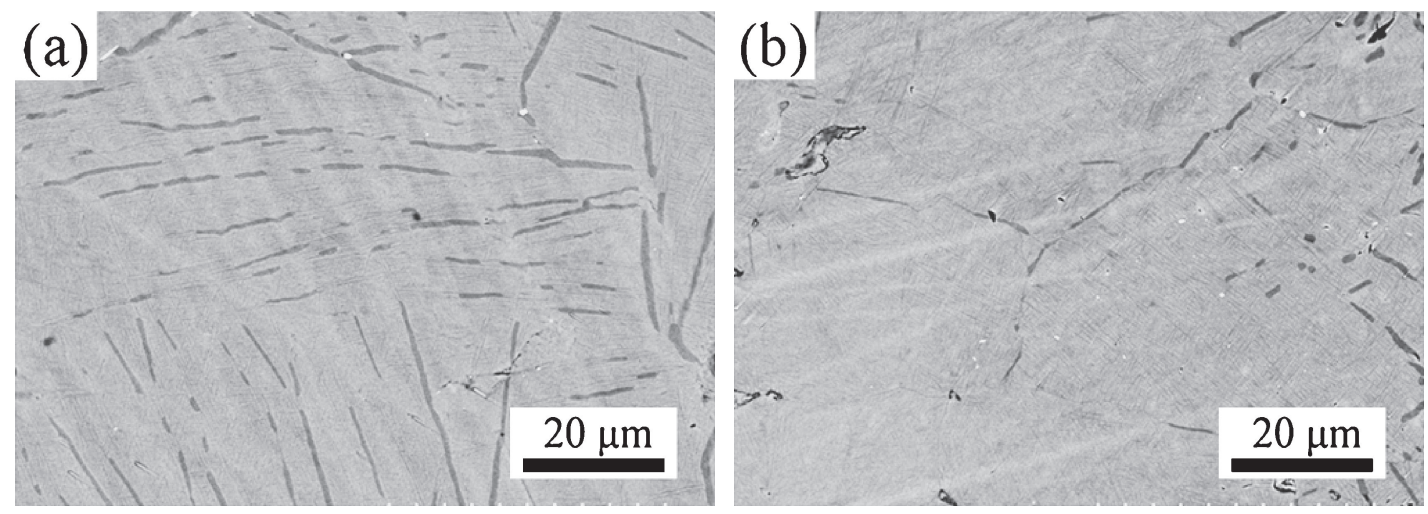

Fig. 2 Microstructrues of the Ti-1.5Fe-2.25Mo alloy heat-treated at $1133 \mathrm{~K}$ for (a) $1 \mathrm{ks}$ and (b) $3.5 \mathrm{ks}$.

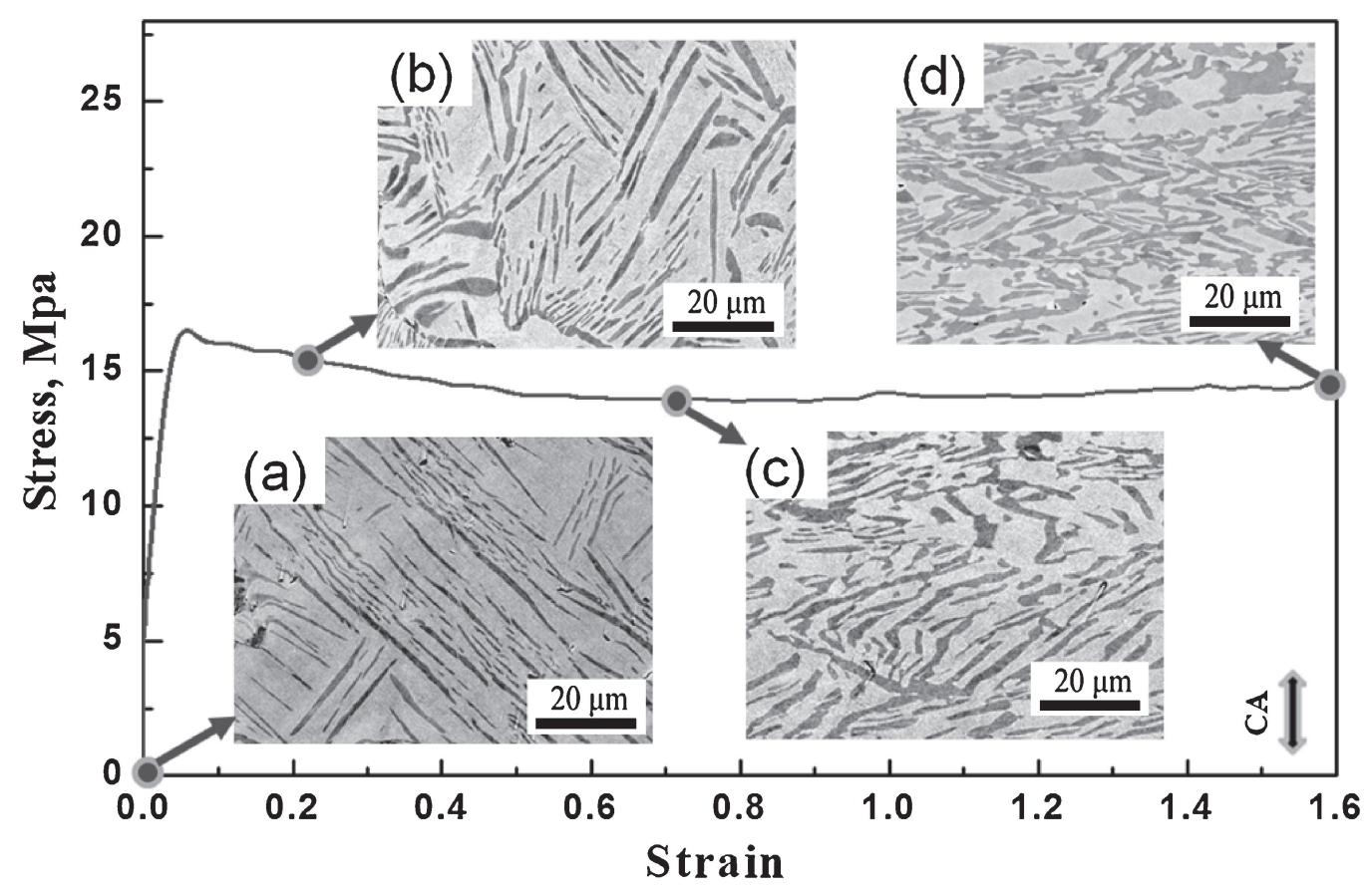

Fig. 3 Flow behavior and microstructural development of the Ti-1.5Fe-2.25Mo alloy during subtransus deformation: (a) $0 \%$, (b) $20 \%$, (c) $50 \%$ and (d) $80 \%$; The effects of friction and work heating on the flow curve were compensated using the procedure given in Ref. 9); CA presents the compression axis.

heat-treatment time increases from 1 to $3.5 \mathrm{ks}$. This is in accordance with that expected from the phase diagram ${ }^{1)}$ where the $\alpha$ phase will transform to the $\beta$ phase as the temperature is increased to $1133 \mathrm{~K}$ (in the $\alpha+\beta$ phase region), and consequently, the volume fraction of the $\alpha$ phase will decrease with time. However, during subtransus hot-deformation, the results show a different tendency. Figure 3 shows the flow curve and the development of microstructure with increasing deformation (the microstructures were observed from the central area of the deformed samples). The flow curve exhibits a flow softening feature, suggesting that certain dynamic softening mechanisms, such as dynamic recrystallization, flow localization, etc., compensate for the hardening effect of deformation. At the initial state of deformation (Fig. 3(a)), the microstructure contains mainly transformed $\beta$ phases and primary $\alpha$ plates (volume fraction approximately $26 \%$ ). As deformation proceeds, as shown in Figs. 3(b)-3(d), the $\alpha$ plates become kinked and the microstructure gets refined, which suggest that the flow softening behavior is caused by the globularization (or dynamic recrystallization) of the lamellar microstructure. However, the volume fraction of the $\alpha$ phase in the deformed samples does not show obvious change as the heat-treated samples does, for example, in the 50\%-deformed and $80 \%$-deformed samples, the volume fractions of the $\alpha$ phase are as high as 27.5 and $29.2 \%$, which are close to that of the undeformed sample. Therefore, the above results suggest that deformation in the subtransus area significantly stabilizes the $\alpha$ phase and restrains the normal $\alpha \rightarrow \beta$ phase transformation.

Figures 4(a) and 4(b) show the TEM bright-field images of the edge and central areas of the $80 \%$-deformed sample, respectively (the observation positions are indicated in Fig. 4(c)). The effective strain distribution in the deformed sample was evaluated by finite element (FE) simulation with a friction coefficient of 0.5 , as shown in Fig. 4(c). The simulated effective strains for the edge and central areas are 0.42 and 2.51, respectively. At the edge low-strain area (Fig. 4(a)), a martensitic $\left(\alpha^{\prime}\right)$ microstructure is observed. 


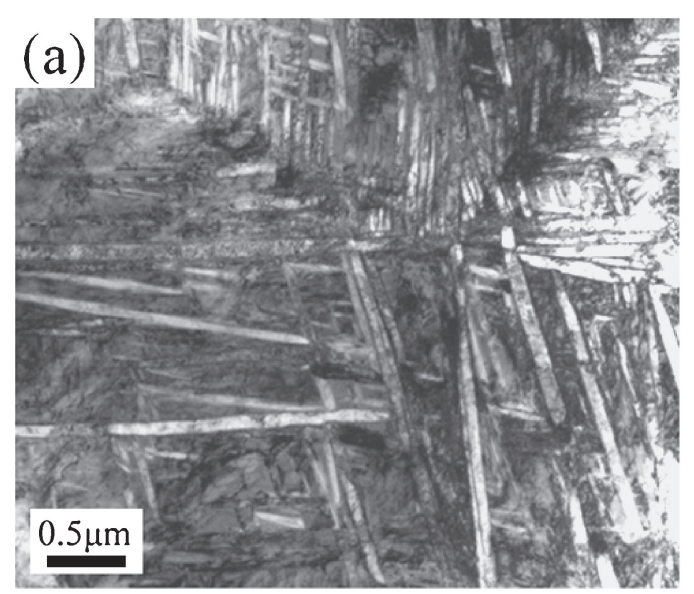

(c)

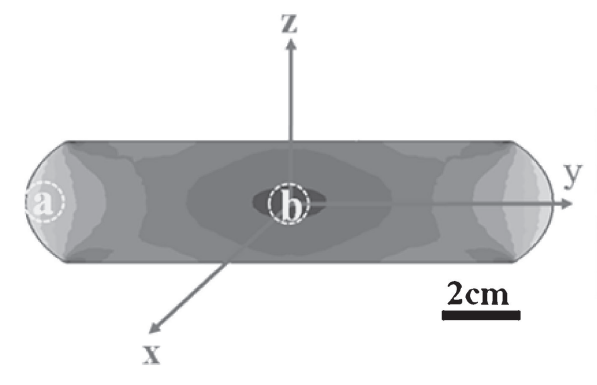

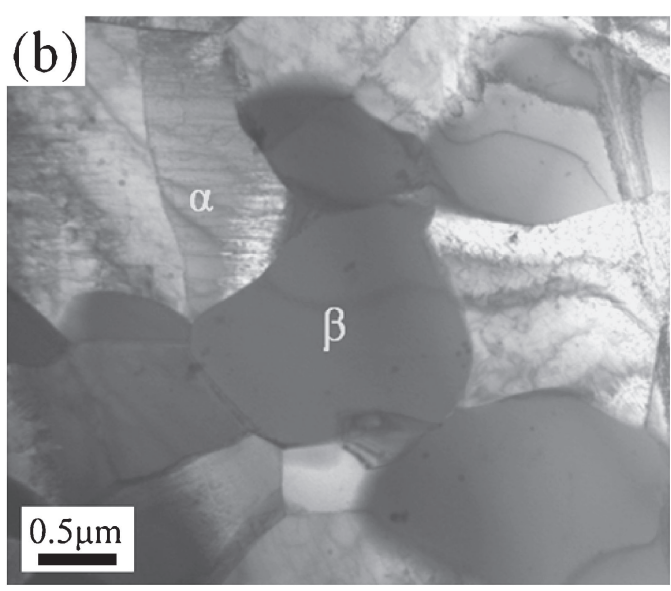

(d)

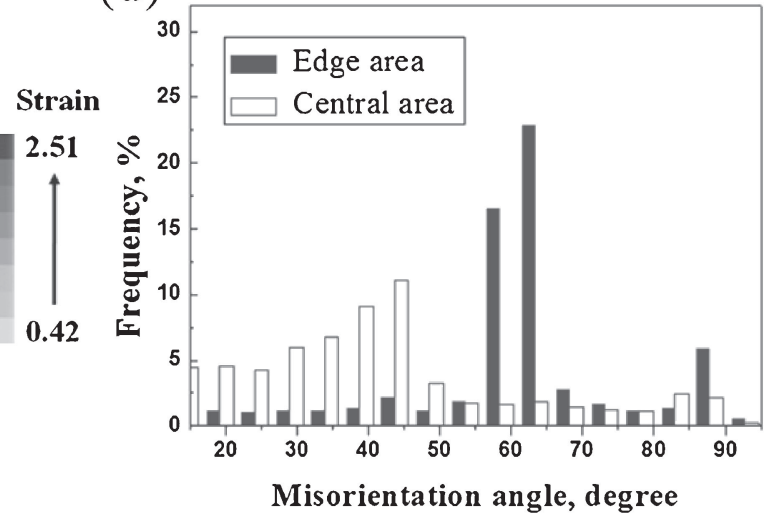

Fig. 4 TEM bright-field images of the (a) edge and (b) central areas of the Ti-1.5Fe-2.25Mo sample deformed to a reduction of $80 \%$; (c) effective strain distribution evaluated from finite element (FE) simulation; (d) grain boundary misorientations distribution.
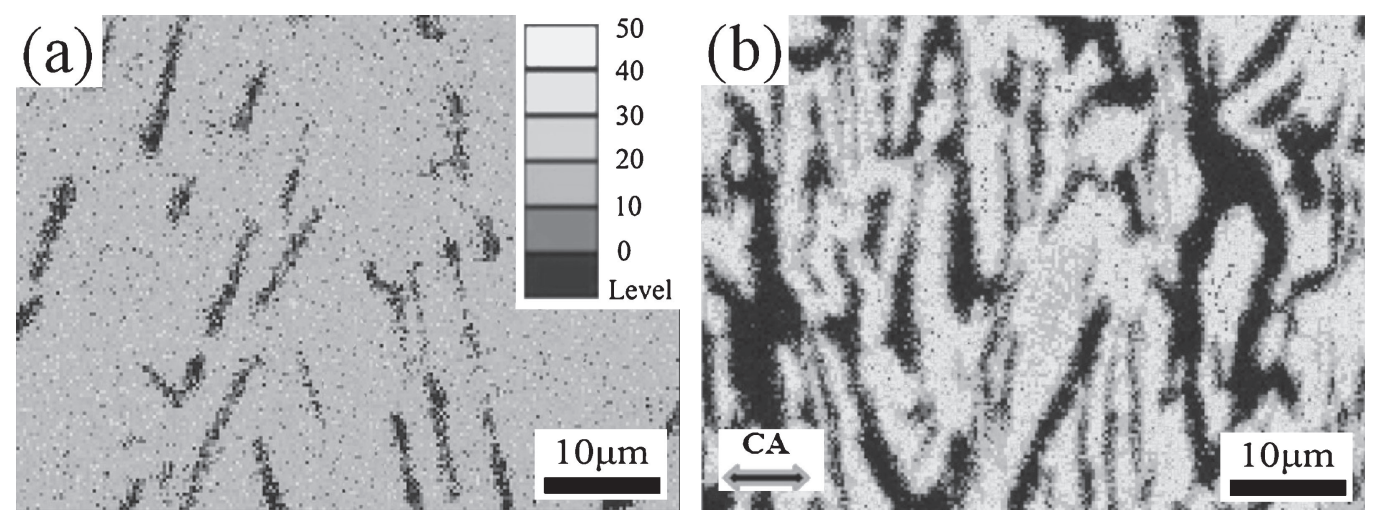

Fig. 5 Composition mapping of element Fe at the (a) edge and (b) central areas of the $80 \%$-deformed sample.

This is confirmed by the grain boundary misorientation distribution (Fig. 4(d)) where an evident peak at about $63.3^{\circ}$ is seen. The boundaries at $63.3^{\circ}$ correspond to the Burgers orientation relationship (OR) derived from the martensitic transformation. ${ }^{10)}$ The formation of the near full martensitic structure indicates that most of the primary $\alpha$ phases are transformed to the $\beta$ phases before cooling. However, at the central high-strain area (Fig. 4(b)), the deformed alloy exhibits an $\alpha+\beta$ binary microstructure, no obvious martensitic transformation can be identified from the grain boundary misorientation, suggesting that a number of primary $\alpha$ phases retain after deformation. Therefore, the above results reveal that there are clear differences in phase structure between the central and edge areas of the deformed pancake, which results from the inhomogeneous deformation. In the high-deformation area, the deformation restrains the $\alpha \rightarrow \beta$ phase transformation and stabilizes the $\alpha$ phase, while in the low-deformation area, this effect is less prominent.

Figures 5(a) and 5(b) show the composition mapping of element $\mathrm{Fe}$ at the edge and central areas of the $80 \%$-deformed sample, respectively. The distribution of element Mo exhibits similar feature as the Fe does, and it is not shown here. The elements $\mathrm{Fe}$ and Mo are enriched almost entirely in the $\beta$ phase because these elements are $\beta$-stabilizing elements, thus, the $\mathrm{Fe}$ and Mo distribution is directly correlated with the $\beta$ 
phase distribution. The absolute contents of Fe and Mo in the $\beta$ phase (on average) at the central area are measured to be 4.8 and 4.1 at\% by SEM-EDS, which are much higher than those at the edge area (1.8 and 1.64 at\%). This high concentration at the central area is caused by the restraint of $\alpha \rightarrow \beta$ phase transformation which inhibits the homogenizing diffusion of the $\mathrm{Fe}$ and Mo. In light of the inhomogeneous distribution of $\mathrm{Fe}$ and $\mathrm{Mo}$, the local martensitic transformation in Fig. 4 appears plausible. It is known that high concentration of $\beta$-stabilizing elements can inhibit martensitic transformation and decrease the martensitic transformation temperature $\left(M_{\mathrm{s}}\right)$ to below room temperature, thereby stabilize the $\beta$ phase to ambient temperature. The critical concentrations for the Fe and Mo are 3.4 and 5.2 at\%, respectively. ${ }^{1)}$ As mentioned before, the concentrations of $\mathrm{Fe}$ and Mo in the $\beta$ phase at the central area are as high as 4.8 and 4.1 at $\%$, which exceed or approach the critical values, therefore, the martensitic transformation in this area is restrained, and the quenched microstructure is characterized by an $\alpha+\beta$ two-phase microstructure. However, at the edge area, the concentrations of $\mathrm{Fe}$ and Mo in the $\beta$ phase are only 1.8 and 1.64 at\%, respectively, which are lower than the critical values, as a result, the martensitic transformation takes place as normal.

The restraint of $\alpha \rightarrow \beta$ transformation during compressive deformation in the present study can be understood in terms of the different atomic packing densities of the two phases. The lattice constants were determined to be $a=0.3295 \mathrm{~nm}$ for the $\beta$ phase and $a=0.293 \mathrm{~nm}$ and $c=0.4675 \mathrm{~nm}$ for the $\alpha$ phase at ambient temperature by using XRD. Given that there are two atoms in $\beta$ unit cell and six atoms in $\alpha$ unit cell, and with the assumption of equivalent packing density, the volume change from $\alpha$ to $\beta$ can be calculated to be $+3.0 \%$. On the other hand, the coefficient of linear thermal expansion of the $\beta$ phase is greater than that of the $\alpha$ phase at the high temperature, ${ }^{11)}$ which also leads to a further increase in the relative volume of the $\beta$ phase. Therefore, the $\alpha \rightarrow \beta$ transformation is accompanied by an obvious volume expansion. The compressive stress might impose a negative factor on the phase transformation with a volume expansion. This might be one of the reasons for the stabilization of $\alpha$ phase under compressive loading.

From the thermodynamic point of view, the free energy change of $\alpha \rightarrow \beta$ transformation without deformation can be expressed by:

$$
\Delta G=-V \Delta G_{\mathrm{c}}+V \Delta G_{\mathrm{s}}+A \gamma_{\alpha / \beta}
$$

where $V$ is the volume, $A$ is the interface area, $\Delta G_{\mathrm{c}}$ is the chemical driving force, $\Delta G_{\mathrm{S}}$ is the volume strain energy, and
$A \gamma_{\beta / \alpha}$ is the interfacial energy. Since the deformation in present study is dominated by the $\beta$ phase, continuous deformation would introduce deformation defects with stored energy within the deformed $\beta$ phases, then, the eq. (1) during deformation can be rewritten as:

$$
\Delta G=-V \Delta G_{\mathrm{c}}+V \Delta G_{\mathrm{s}}+A \gamma_{\beta / \alpha}+V \Delta G_{\mathrm{d}}
$$

where $\Delta G_{\mathrm{d}}$ is the deformation stored energy. Therefore, the excess stored energy, $\Delta G_{\mathrm{d}}$, would influence the phase equilibrium and act as a resistance for the $\alpha \rightarrow \beta$ transformation. In addition, the strain energy, $\Delta G_{\mathrm{s}}$, induced by the $\alpha \rightarrow \beta$ transformation would increase under the compressive loading, which is also unfavorable for the $\alpha \rightarrow \beta$ transformation. The detailed mechanism responsible for the restraint of $\alpha \rightarrow \beta$ phase transformation is still unclear and more detailed theoretical and experimental studies are now being conducted and will be reported later.

\section{Conclusion}

In summary, dynamic phase transformation behavior of an $\alpha+\beta$ type Ti-1.5Fe-2.25Mo alloy during subtransus deformation was investigated. The results show that compressive deformation restrains the $\alpha \rightarrow \beta$ transformation in the $\alpha+\beta$ phase region and leads to a local martensitic transformation in the deformed sample during cooling. This insight into hot forging provides a new pathway for controlling the microstructure of engineering titanium alloys.

\section{REFERENCES}

1) G. Lütjering and J. C. Williams: Titanium: Engineering Materials and Processes, 2nd ed., (Springer-Verlag Berlin, 2007) pp. 29-33.

2) T. Ahmed and H. J. Rack: Mater. Sci. Eng. A 243 (1998) 206-211.

3) C. Loier and G. Thauvin: J. Less Common Metals 108 (1985) 295-312.

4) J. Koike, Y. Shimoyama, I. Ohnuma, T. Okamura, R. Kainuma, K. Ishida and K. Maruyama: Acta Mater. 48 (2000) 2059-2069.

5) H. S. Yang, G. Gurewitz and A. K. Mukherjee: Mater. Trans. JIM 32 (1991) 465-472.

6) K. L. Yang, J. C. Huang and Y. N. Wang: Acta Mater. 51 (2003) 25772594.

7) S. L. Semiatin, V. Seetharaman and I. Weiss: Mater. Sci. Eng. A 243 (1998) 1-24.

8) S. Tamirisakandala, B. V. Vedam and R. B. Bhat: J. Mater. Eng. Perform. 12 (2003) 661-673.

9) Y. P. Li, E. Onodera, H. Matsumoto and A. Chiba: Metall. Mater. Trans. A 40 (2009) 982-990.

10) S. Mironov, M. Murzinova, S. Zherebtsov, G. A. Salishchev and S. L. Semiatin: Acta Mater. 57 (2009) 2470-2481.

11) J. F. Shackelford and W. Alexander: Materials Science and Engineering Handbook, 3rd ed., (CRC Press LLC, New York, 2001) pp. 461466. 